\title{
Immunodeficiency due to defective antigen processing: the molecular basis for type 1 bare lymphocyte syndrome
}

\author{
Malini Raghavan \\ Department of Microbiology and Immunology, 5641 Medical Science Building II, \\ University of Michigan Medical School, Ann Arbor, Michigan 48109-0620, USA
}

Address correspondence to: Department of Microbiology and Immunology, 5641 Medical Science Building II, University of Michigan Medical School, Ann Arbor, Michigan 48109-0620, USA. Phone: (734)647-7752; Fax: (734)764-3562; E-mail: malinir@umich.edu

Major histocompatibility complex (MHC) class I molecules are polymorphic cell surface glycoproteins that play an essential role in immune surveillance. In the MHC class I antigen presentation pathway, peptides derived from cytosolic proteasome-mediated proteolysis are transported into the endoplasmic reticulum (ER) via the transporter associated with antigen processing (TAP), reviewed in ref. 1. In the ER lumen, translocated peptides bind to newly synthesized complexes of MHC class I heavy chains and $\beta 2$-microglobulin $(\beta 2 \mathrm{~m})$. Binding is facilitated by the transient association of class I heterodimers and TAP, an interaction that is bridged by the ER-resident protein tapasin (Fig. 1 and ref. 2). Pep- tide binding to MHC class I molecules is believed to result in structural changes that lead to the dissociation of peptideMHC complexes from TAP complexes and tapasin, and their subsequent transit to the cell surface, reviewed in ref. 1. Cell surface display of foreign peptides in complex with MHC class I molecules allows for $\mathrm{CD}^{+} \alpha \beta \mathrm{T}$ cell-mediated recognition of cells that bear intracellular pathogens.

TAP comprises two structurally related subunits, which interact to form a functional peptide-transporting complex (Fig. 1). Prior to peptide translocation by TAP, peptides bind to the membrane-proximal, cytosolic surface of TAP1/TAP2 complexes (3). Hydrolysis of ATP results in peptide translocation into the ER lumen, reviewed in ref. 1. Studies with mutant mammalian cell lines have shown that both TAP1 and TAP2 are required for peptide binding to TAP (3), as well as for peptide translocation into the ER (4). Defects in either protein result in an impairment in peptide translocation, and in the absence of class I-specific peptides in the ER. MHC class I molecules that are not complexed with peptides are unstable, inefficiently transported through the Golgi, and poorly expressed at the cell surface, reviewed in ref. 5. Cells derived from mutant mice with a disrupted TAP1 gene show highly reduced levels of surface MHC class I molecules, and are also

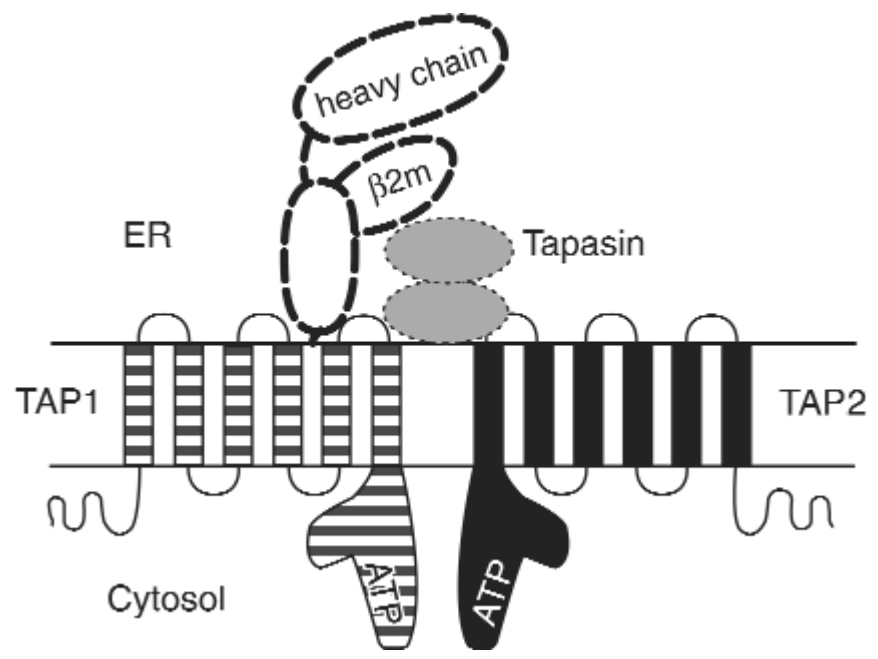

Figure 1

Selected components of the MHC class I antigen presentation pathway. The structures of individual components, modes of interaction between the different components, and stoichiometries of binding remain to be fully defined. TAP1 (solid line, striped) and TAP2 (solid line, black) each contain multiple membrane-spanning segments and C-terminal ATP-binding domains, depicted here as L-shaped structures, based upon the crystal structure of analogous domains of a bacterial histidine transporter (15). TAP1 and TAP2 interact to form a functional peptide transporting complex. In the ER lumen, translocated peptides associate with MHC class I heavy chains and $\beta 2 \mathrm{~m}$ (bold dashed lines) (16). Peptide binding by MHC class I molecules is facilitated by the transient formation of TAP-MHC class I complexes. This interaction involves tapasin (dotted lines, gray) as well as calreticulin (2), a cellular chaperone (not shown in the figure). Tapasin interactions with MHC class I molecules may resemble class I interactions with CD8, a coreceptor for the T-cell receptor (17). Defects in either TAP1 or TAP2 result in defective assembly of class I heterodimers and have been shown to cause human MHC class I deficiencies $(7,9,10)$. It is likely that other cases of MHC class I deficiencies will map to mutations that interfere with the stable expression of additional components of the MHC class I antigen presentation pathway, or with interactions between the different components. 
significantly impaired in the development of mature $\mathrm{CD}^{+} \alpha \beta$ T cells (6).

Inherited human deficiencies in the TAP2 subunit have previously been reported in a few patients with MHC class I deficiency, termed the type I bare lymphocyte syndrome (BLS) (7). The most significant effect of TAP2-deficiency in humans appears to be the onset of chronic bacterial colonization of the respiratory tract, observed when patients reach late childhood (7). Analysis of MHC class I expression levels in peripheral blood mononuclear cells (PBMCs) from TAP2-deficient patients indicated a severe reduction in cell surface protein ( $1 \%$ to $3 \%$ of normal individuals), while mRNA levels were unaffected (7). The expression levels of the class Ib molecule CD1a on epidermal Langerhan's cells of the patients were unaffected, consistent with the lack of requirement of the TAP transporter for maturation and surface expression of CD1 (8). Mature $\mathrm{CD}^{+} \alpha \beta$ $\mathrm{T}$ cells were found in significant numbers in PBMCs from TAP2-deficient individuals, although at reduced levels compared to normal individuals (7). It was suggested that a more extensive exposure to pathogens might account for the increased numbers of mature $\mathrm{CD}^{+} \alpha \beta$ T cells in TAP-deficient humans compared to laboratory-bred, TAP-deficient mice (7).

In this issue of the Journal of Clinical Investigation, Furakawa et al. (9) and de la Salle et al. (10) report the first examples of human TAP1 deficiencies in patients with BLS. Both groups studied cells derived from a Japanese patient, first reported by Maeda et al. (11) as a case of bare lymphocyte syndrome in which disease was characterized by late childhood onset of chronic sino-bronchial infections, as well as skin lesions of unknown cause that developed at a later age. In addition, de la Salle et al. studied cells derived from an Italian patient with defective expression of MHC class I molecules. Both patients are shown to have a genetic defect in the TAP1 gene, which leads to the absence of a functional TAP1 subunit. Interestingly, the expression of the TAP2 subunit is also impaired, although no mutations were identified in the TAP2 gene (9). Thus, under some conditions, TAP1 may be required for maintaining stable expression of TAP2. As expected, the absence of functional TAP subunits results in reduced cell surface expression of MHC class I molecules in fibroblasts and Epstein Barr virus-transformed B cell lines derived from the two patients. Cell-surface expression of MHC class I molecules could be partially restored by the addition of exogenous peptides of appropriate specificity. In addition, de la Salle et al. demonstrate that vaccinia virus-mediated expression of TAP1, but not TAP2, can restore MHC class I expression in cells derived from the TAP1-deficient patients. Furakawa et al. infer a lack of tapasin association with class I heavy chains in cells that lack the TAP proteins. However, this observation is contrary to results from previous studies with TAPdeficient cells, in which tapasin-class I heavy chain associations have been documented [for example, (2)]. The maturation of $\mathrm{CD}^{+} \mathrm{T}$ cells in the TAP1-deficient individuals remains to be described, and will allow important comparisons with TAP2-deficient individuals as well as with TAP1-deficient mice.

Consistent with the predominant view that both TAP subunits are required for peptide translocation from the cytosol into the ER, the reports by Furakawa et al. (9) and de la Salle et al. (10) show that the clinical manifestations of TAP1 deficiency are, for the most part, similar to the previously characterized TAP2 deficiency (7). Of particular interest is the observation that neither the TAP1- nor the TAP2-deficient individuals suffer from chronic viral infections. High titers of antibodies against several viruses were detected in the serum of TAP2-deficient individuals, suggesting that antibodymediated responses might play a compensatory role in anti-viral defense of these patients (7). The observation of chronic bacterial lung infections in TAP1- and TAP2-deficient patients also suggests that presentation of bacterial antigens by MHC class I molecules on lung macrophages (13) may represent a physiologically important mechanism for the control of bacterial pathogens.

It is conceivable that other defects in known components of the MHC class I antigen presentation pathway might result in immunodeficiencies that resemble TAP1 or TAP2 deficiencies (Fig. 1). Furthermore, it is likely that other unidentified proteins are essential components of the class I antigen presentation pathway, and function, for example, in the sequestration and targeting of peptides to the ER membrane (14). Future studies of the cell biology and biochem- istry of MHC class I antigen presentation will result in the identification of such components. In addition, analyses of the molecular basis of other cases of human MHC class I deficiencies will allow for correlations between the occurrence of disease, and the presence of mutations in newly discovered components of the class I antigen presentation pathway.

1. Momburg, F. and Hämmerling,G. 1998. Generation and TAP-mediated transport of peptides for major histocompatibility complex class I molecules. Adv. Immunol. 68:191-256.

2. Sadasivan, B., Lehner P. J., Ortmann, B., Spies, T. and Cresswell, P. 1996. Roles for calreticulin and a novel glycoprotein, Tapasin, in the interaction of MHC class I molecules with TAP. Immunity. 5:103-114.

3. Androlewicz, M. J., Ortmann, B., van Endert,P., Spies, T., and Cresswell, P. 1994. Characteristics of peptide and major histocompatibility complex class $\mathrm{I} / \beta 2$-microglobulin binding to the transporters associated with antigen processing (TAP1 and TAP2). Proc. Natl. Acad. Sci. USA. 91:12716-12720.

4. Kelly, A., et al. 1992. Assembly and function of the two $\mathrm{ABC}$ transporter proteins encoded in the human major histocompatibility complex. Nature. 355:641-644.

5. Heemels, M.-T. and Ploegh, H. 1995. Generation, translocation, and presentation of MHC class Irestricted peptides. Ann. Rev. Biochem. 64:463-491.

6. Van Kaer, L., Ashton-Rickardt, P.G., Ploegh, H. and S. Tonegawa. 1992. TAP1 mutant mice are deficient in antigen presentation, surface class I molecules and CD4-8+ T cells. Cell. 71:1205-1214.

7. de la Salle, H., et al 1994. Homozygous human TAP peptide transporter mutation in HLA class I deficiency. Science. 265:237-240.

8. Sugita, M., Porcelli, S.A., and Brenner, M.B. 1997. Assembly and retention of Cd1b heavy chains in the endoplasmic reticulum. J. Immunol. 159:2358-2365.

9. Furakawa, H., et al. 1999. Splice acceptor site mutation of the transporter associated with antigen processing 1 gene in human histocompatibility leukocyte antigen class I deficiency. J. Clin. Invest. 103:755-758

10. de la Salle, H., et al. 1999. HLA class I deficiencies due to mutations in the subunit 1 of the peptide transporter (TAP1). J. Clin. Invest. 103:R9-R13.

11. Maeda, H., et al. 1985. Defective expression of HLA class I antigens: a case of bare lymphocyte without immunodeficiency. Immunogenetics 21:549-558.

12. Plebani, A., et al. 1996. Defective expression of HLA class I and CD1a molecules in a boy with Marfan-like phenotype and deep skin ulcers. $J$. Am. Acad. Dermatol. 35:814-818.

13. Pfeifer, J.D., et al. 1993. Phagocytic processing of bacterial antigens for class I MHC presentation to T cells. Nature. 361:359-362.

14. Deng, Y., et al. 1998. Assembly of MHC class I molecules with biosynthesized endoplasmic reticulum-targeted peptides is inefficient in insect cells and can be enhanced by protease inhibitors. J. Immunol. 161:1677-1685.

15. Hung, L.-W., et al. 1998. Crystal structure of the ATP-binding subunit of an $\mathrm{ABC}$ transporter. Nature. 396:703-706.

16. Bjorkman, P. J., et al. 1987. Structure of the human class I histocompatibility antigen, HLA-A2. Nature. 329:506-512.

17. Suh, W.-K., et al. 1999. Interaction of murine MHC class I molecules with tapasin and TAP enhances peptide loading and involves the heavy chain $\alpha 3$ domain. J. Immunol. 162:1530-1539. 